\title{
Spectrum of Cytological Findings In Patients with Superficial Lymphadenopathy: A Five Year Retrospective Study
}

\author{
Authors \\ Dr Rajesh Gaur, Dr Poonam Woike \\ Department of Pathology, G.R Medical College \& J.A Group of Hospitals, Gwalior, India \\ Corresponding Author \\ Dr. Poonam Woike \\ \#35, Senior Girls Hostel J.A Hospital Campus, Lashkar, Gwalior, India 474009 \\ Email id-poonam.woike@gmail.com Phone no- 7745983670
}

\begin{abstract}
Superficial lymphadenopathy is ranked among the most common clinical findings encountered in medical practice. Fine needle aspiration cytology $(F N A C)$ is a veritable tool for the assessment and diagnosis of superficial lymph node enlargement. The aim of our study is to see the profile of superficial lymphadenopathy in our region and the role of FNAC in the definite diagnosis of cause of lymphadenopathy. In this 5 years study a total of 1188 patients with superficial lymphadenopathy were referred from the out patients clinics for cytological examination. FNAC was performed using 23 gauge needle attached to $20 \mathrm{ml}$ syringe. Aspirated materials were smeared into 2-3 slides which were stained with May Grunwald Giemsa (MGG) stain and using Dibutyl Phathalate Xylene (D.P.X) mountant were prepared for cytological examination. Five year data was collected and compiled into various categories. The cervical region was involved in most of the cases (90.15\%) followed by the axillary region (6.98\%) and lastly by inguinal region (2.3\%). Tubercular lymphadenitis (53.9\%) was the commonest cause of lymphadenopathy followed by reactive lymphoid hyperplasia (28.8\%), metastasis (13.4\%) and lymphomas (2.86\%). Squamous cell carcinoma (9.5\%) followed by adenocarcinoma (3.9\%) were the most frequent metastatic tumors. FNAC is a useful diagnostic tool in the management of patients presenting with lymphadenopathy and should be considered before more invasive and costly procedures are performed, particularly in developing countries.

Keywords: Lymphadenopathy, FNAC, Diagnosis, Malignancy.
\end{abstract}

\section{Introduction}

Lymphadenopathy or lymphadenitis refers to lymph nodes which are abnormal in size, number or consistency. Lymph nodes are the easily accessible components of lymphoid tissue that not only clean and filter lymph but also produce lymphocytes and antibodies. The human body has approximately 600 lymph nodes, some of which submandibular, axillary or inguinal lymph nodes may normally be palpable in healthy people. 
Lymphadenopathy refers to nodes that are abnormal in size, consistency or number (Darnal et al., 2005 ${ }^{[1]}$. It is one of the commonest and significant clinical presentations of patients, attending the outdoor clinics in most hospitals. It can be of two types- local due to a localizes spot of infection eg., an infected spot on scalp will cause lymph nodes in the neck on that same side to swell up, generalized due to systemic infection of the body e.g., influenza or secondary syphilis and dermatopathic lymphadenopathy which is associated with skin diseases. Types are reactive (due to acute or chronic infections), tumoral (Hodgkin and non-hodgkin lymphoma), autoimmune (SLE, rheumatoid arthritis) and immunocompromised (HIV infection).

FNAC is an easy, safe, reliable, rapid and inexpensive method for diagnosing enlarged lymph nodes with a high degree of accuracy. Typically in humans, lymphadenopathy is superficially or deeply located. Superficial lymphadenopathy ranked among the most common clinical findings encountered in the etiology of lymph nodes enlargement range from spectrum of infections, reactive hyperplasia to malignant diseases. Diagnosing these lesions poses a major challenge to the clinicians. However fine needle aspiration cytology (FNAC) has become a authentic tool for assessment and diagnosis of 2 superficial lymph node enlargement. Studies have shown that the diagnostic lymph node enlargement sensitivity and specificity of FNAC is $90 \%$ and $95 \%$ respectively. In this study, the most common anatomical site for the superficial lymphadenopathy was the cervical group of lymph nodes. This constituted $32.9 \%$ of all anatomical sites. This was closely followed by axillary group of lymph nodes accounting for $16.4 \%$ and lastly inguinal region. This again, is in close relation to reports by other ${ }^{[2,3]}$ researchers in other part of the globe. Among the inflammatory lesions tuberculosis (TB) is one of the most common infective causes of superficial lymphadenopathy in developing countries $^{[2,16]}$ including Nigeria.

\section{Materials and methods}

The study was conducted in cytopathology section of Department Of Pathology of our centre. Patients who visited the OPD of our hospital, with complaints of superficial lymphadenopathy were sent for FNAC for proper diagnosis.informed consent was taken from the patients. FNAC was performed using 23 gauge needle attached to 20 $\mathrm{ml}$ syringe. In each procedure, an average of 2 to 3 passes is usually performed and aspirated materials smeared into 2-3 slides. Slides were stained with May Grunwald Giemsa (MGG) stain and using D.P.X mountant were prepared for cytological examination. The reporting was done by experienced cytologists and the data of reported cases over past five years (2010-2014) were collected and categorized. The data from the 2010-2014 were retrieved, compiled, summarized and statistically analyzed by frequency distribution and percentage proportion.

Limitations of the study- the study could only be carried out in the patients who visited our centre, so the actual profile in this region was not ruled out due to the undiagnosed cases in this region.

\section{Results}

In this 5 years study a total of 1188 patients with superficial lymphadenopathy were examined. In our study, the most common superficial lymphadenopathy is cervical $(90.15 \%)$, followed by axillary $(6.98 \%)$ and lastly inguinal $(2.3 \%)$. Following tables show the profile of lymphadenopathy in various anatomical positions, age groups and different cytological diagnosis after the examination (Table No. 1, 2, 3, 4 And Fig No. 1 \& 2). 


\section{JMSCR Vol||3||Issue||10||Page 7933-7939||October}

Table No. 1.Year Wise Distribution Of Lymphadenopathy-

\begin{tabular}{|l|c|c|c|c|}
\hline Year & Cervical & Axillary & Inguinal & Total \\
\hline 2010 & $190(81.54 \%)$ & $35(15.02 \%)$ & $08(3.4 \%)$ & 233 \\
\hline 2011 & $160(68.66 \%)$ & $20(10.8 \%)$ & $05(2.7 \%)$ & 185 \\
\hline 2012 & $230(95 \%)$ & $10(4.1 \%)$ & $02(0.8 \%)$ & 242 \\
\hline 2013 & $243(94 \%)$ & $12(4.65 \%)$ & $03(1.16 \%)$ & 258 \\
\hline 2014 & $248(95.3 \%)$ & $06(2.30 \%)$ & $05(1.9 \%)$ & 260 \\
\hline
\end{tabular}

Table No.2.Age Wise Distribution Of Lympadenopathy -

\begin{tabular}{|c|c|c|c|}
\hline Year & $0-15$ Years & $16-45$ Years & $>45$ Years \\
\hline 2010 & $45(19.31 \%)$ & $128(54.9 \%)$ & $60(25.75 \%)$ \\
\hline 2011 & $40(21.6 \%)$ & $105(56.75 \%)$ & $45(24.32 \%)$ \\
\hline 2012 & $52(21.48 \%)$ & $145(59.9 \%)$ & $50(20.66 \%)$ \\
\hline 2013 & $53(20.54 \%)$ & $147(56.97 \%)$ & $58(22.48 \%)$ \\
\hline 2014 & $56(21.53 \%)$ & $152(58.1 \%)$ & $52(20 \%)$ \\
\hline
\end{tabular}

Table No. 3.Distribution Of Findings In Various Lymphadenopathy-

\begin{tabular}{|l|l|l|l|l|l|}
\hline Year & Tuberculosis & Reactive & $\begin{array}{l}\text { Metastasis Sqamous } \\
\text { Cell Carcinoma }\end{array}$ & $\begin{array}{l}\text { Metastasis } \\
\text { Adenocarcinoma }\end{array}$ & Lymphoma \\
\hline 2010 & $120(51.5 \%)$ & $74(31.7 \%)$ & $25(10.7 \%)$ & $06(2.5 \%)$ & $08(3.4 \%)$ \\
\hline 2011 & $107(57.8 \%)$ & $50(27 \%)$ & $16(8.6 \%)$ & $07(3.7 \%)$ & $05(2.7 \%)$ \\
\hline 2012 & $135(55.7 \%)$ & $70(28.9 \%)$ & $23(9.5 \%)$ & $08(3.3 \%)$ & $06(2.4 \%)$ \\
\hline 2013 & $138(53.4 \%)$ & $74(28.6 \%)$ & $25(9.6 \%)$ & $12(4.6 \%)$ & $09(3.4 \%)$ \\
\hline 2014 & $141(54.2 \%)$ & $75(28.8 \%)$ & $24(9.2 \%)$ & $14(5.3 \%)$ & $06(2.3 \%)$ \\
\hline
\end{tabular}

Table No. 4. Male/ Female Distribution-

\begin{tabular}{|l|c|c|}
\hline Year & No. Of Males & No. Of Females \\
\hline 2010 & $144(61.8 \%)$ & $89(38.19 \%)$ \\
\hline 2011 & $120(64.8 \%)$ & $65(35.1 \%)$ \\
\hline 2012 & $135(55.7 \%)$ & $107(44.2 \%)$ \\
\hline 2013 & $143(55.4 \%)$ & $115(44.5 \%)$ \\
\hline 2014 & $157(60.3 \%)$ & $103(39.6 \%)$ \\
\hline
\end{tabular}

Anatomical Distribution Of Lymphadenopathy

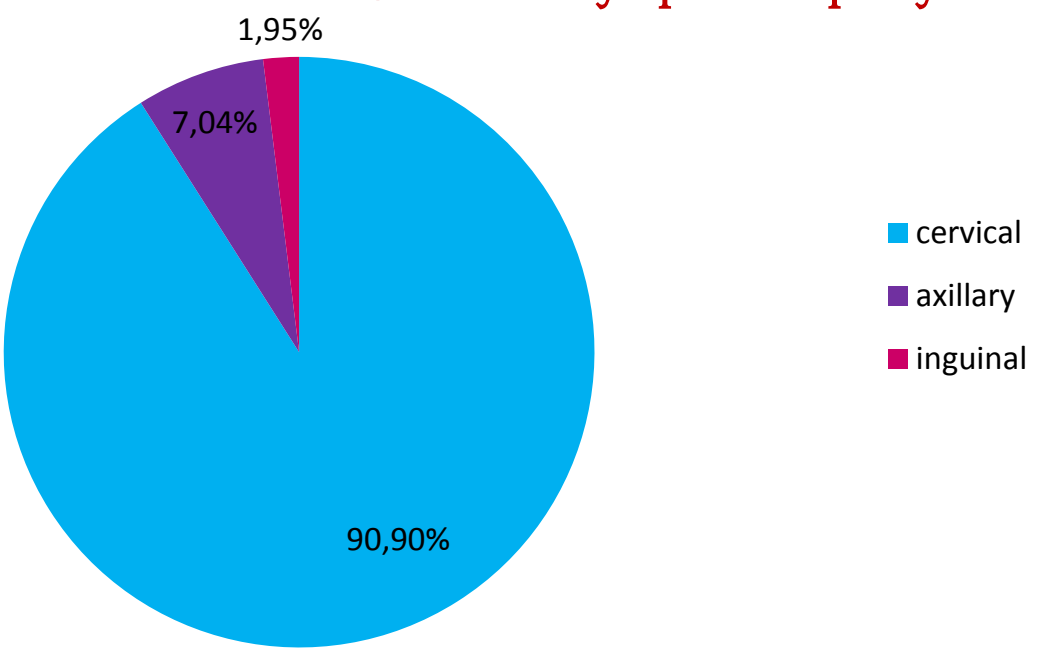

Fig no. 1. Anatomical Distribution Of Superficial Lymphadenopathy Reported From 2010-2014 


\section{Various Lesions Diagnosed In Different Lymphadenopathy}

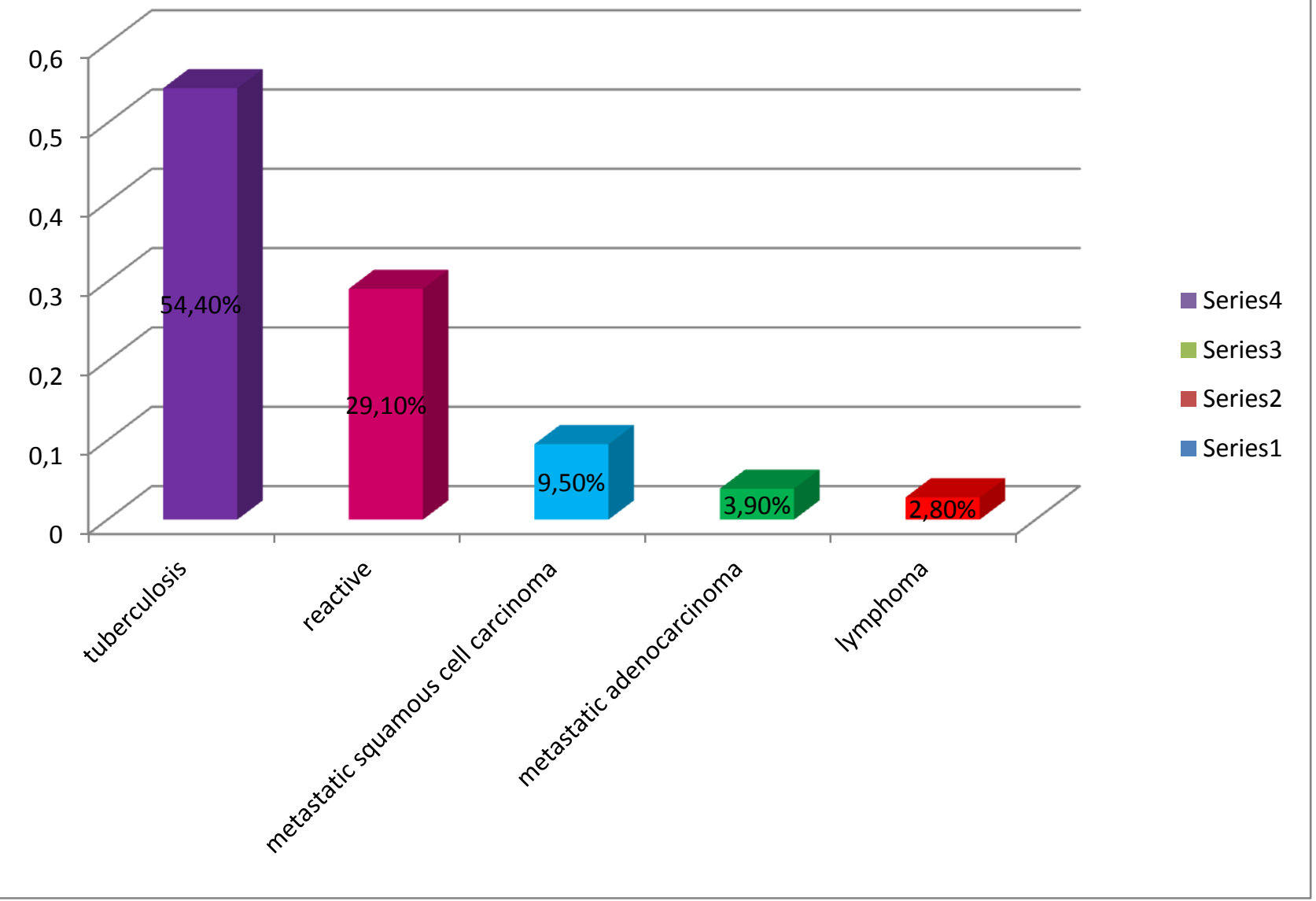

Fig No.2. Final Diagnosis Of Lesions After FNAC.

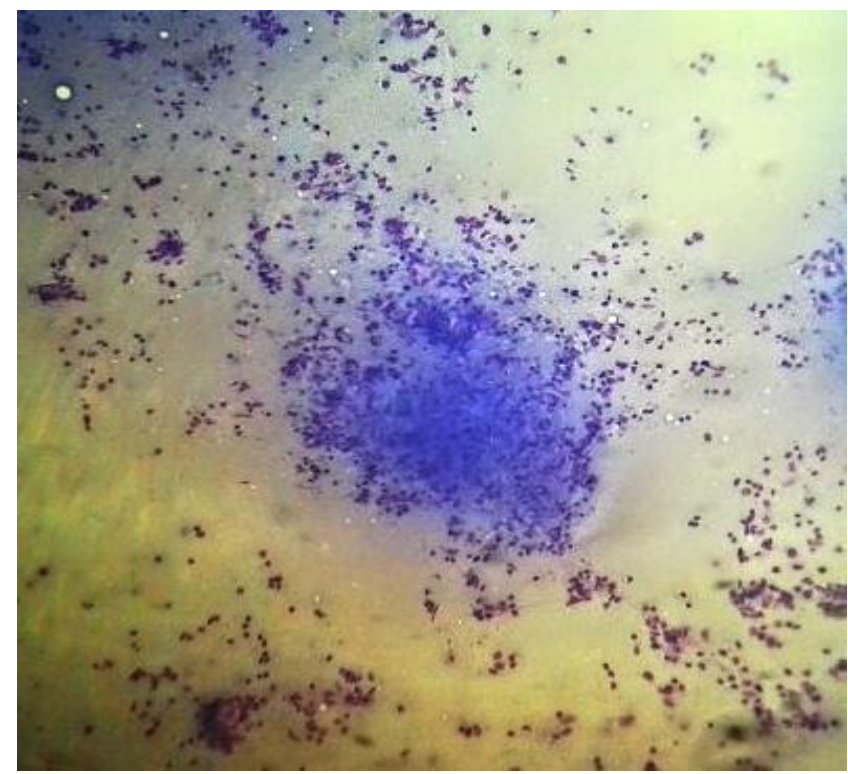

Figure showing granuloma formation MGG stain $10 \mathrm{X}$

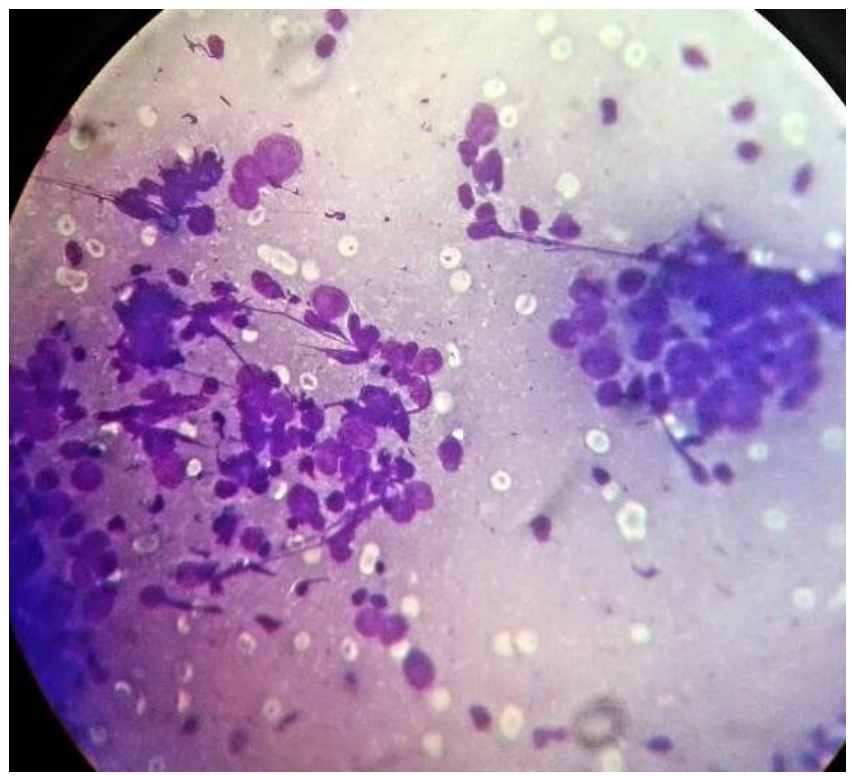

Figure showing metastatic deposits of squamous cell carcinoma MGG stain 40x 


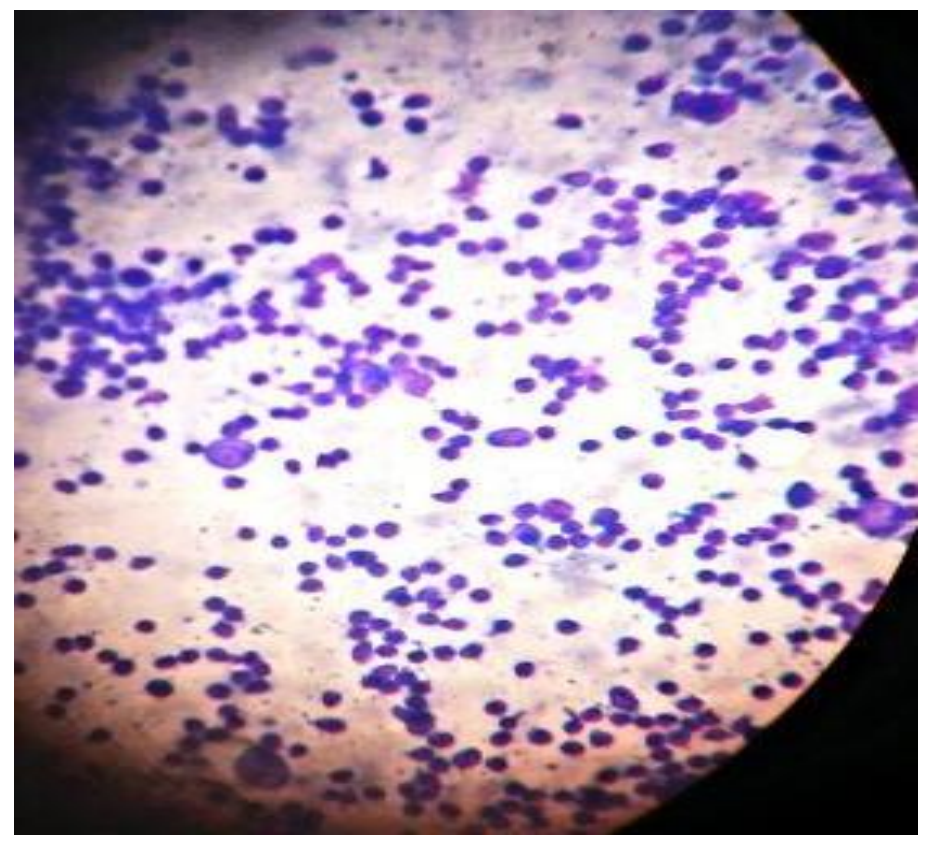

Figure showing reactive lymphadenopathy MGG stain $10 \mathrm{X}$

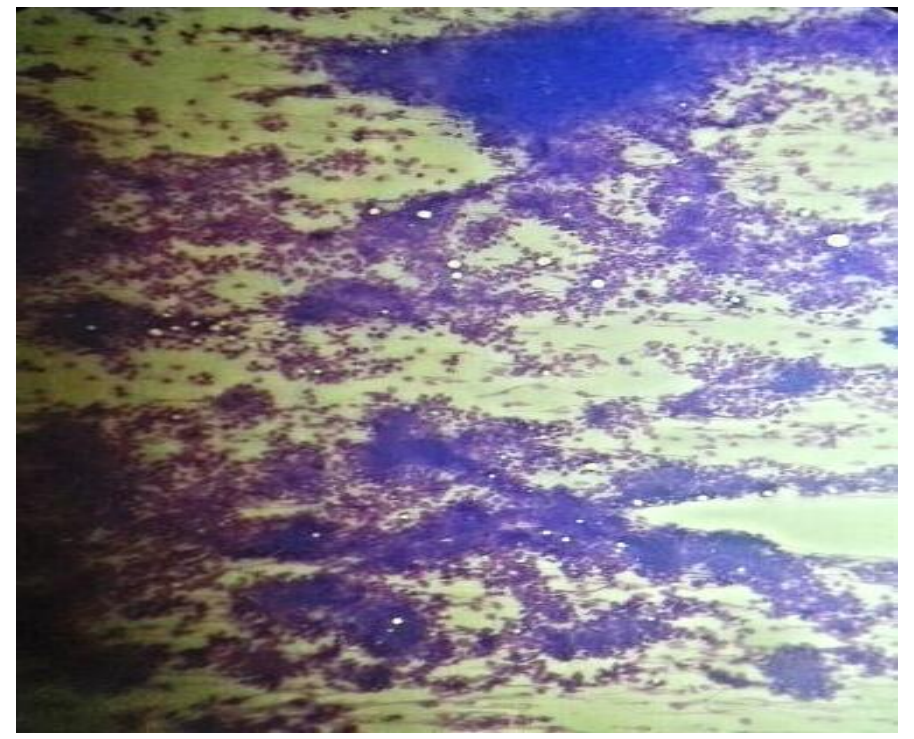

Figure showing non-hodgkin's lymphoma MGG stain $10 \mathrm{X}$

\section{Discussion}

Lymphadenopathy is a clinical manifestation of regional or systemic disease which serves as an excellent clue to the underlying disease (Bhuyan et al., 2008 ${ }^{[4]}$. It can arise either from benign or malignant causes depending upon the geographical condition and socioeconomic set up (Ahmad et al., 2009)[5]. In this study, a total number of 1188 cases of lymphadenopathy were studied out of which $82.82 \%$ were benign and $16.32 \%$ were malignant. Ahmad et al., 2005 ${ }^{[5]}$, showed benign causes contributed to $86.4 \%$ of lymphadenopathies. Studies from other developing countries like Pakistan (Khan et al., 2011; Fatima et al., 2011) ${ }^{[6,7]}$ also reported $92 \%$ and $73.2 \%$ benign lymphadenopathies. In one study from Nepal, benign lesions were reported in $81.5 \%$ cases of lymphadenopathy compared to only $18.5 \%$ of malignant lesions. Nevertheless, this is at variance with report by Steel et $\mathrm{al}^{[8]}$ where malignant and benign lesions accounted for $59 \%$ and $34 \%$ respectively. In our study, metastatic carcinoma (squamous cell carcinoma and adenocarcinoma) accounted for $10.9 \%$ of all cases. This is in keeping with reports by Fatima et al ${ }^{[8]}$ where metastatic carcinoma accounted for $8.5 \%$ of the lesions. Squamous cell carcinoma $(32.2 \%)$ followed by adenocarcinoma (22\%) were the most common metastatic tumors in our study. Similar figures were also seen in a study (Mitra et al., 2011) ${ }^{[9]}$. Other studies (Khajuria et al., 2006; Hirachand et al., 2009; Wilkinson et al., 2012) ${ }^{[10-}$ 12] also found squamous cell carcinoma as the most common metatatic malignancy. The most common age group which presented with lymphadenopathy was between 16-45 years of age, followed by $>45$ years and $0-5$ years. Out of the total 1188 lymphadenopathies reported $90.15 \%$ were cervical , $6.98 \%$ axillary and $23 \%$ inguinal. Many others (Pandit et al., 1987; Ahmad et al., 2005; Nidhi et al., 2011) ${ }^{[13,5,14]}$ also found cervical region to be the most common site of involvement. After categorization of different findings in various age groups it was seen that in the age group 0-5 years maximum cases had reactive lymphadenitis followed by tubercular lymphadenitis and lymphomas (hodgkins and nonhodgkins), another study (Dhingra et al., 2010) ${ }^{[15]}$ showed reactive lymphadenopathy as the most common cause followed by granulomatous lymphadenitis. Metastatic carcinoma was nil this age group. In 16-45 years maximum cases presented with tubercular lymphadenitis followed by reactive lymphadenitis, lymphomas and lastly metastatic carcinoma, >45 years presented maximally with metastatic deposits of carcinoma, 
followed by tubercular lymphadenitis, reactive and lymphomas. The male and female ratio was found to be 1.42:1. It can be noted from the above data that the number of patients coming for FNAC for superficial lymphadenopathy increased from 2010 to 2014 which signifies the increase in awareness of people in our region, which leads to the early diagnosis of various conditions and proves beneficiary to the patients. Also, the cases positive for tuberculosis increased from 120 to 160 in the last five years (i.e from 2010-2014) which correspond to the disease burden in this area, hence FNAC proves a reliable diagnostic criteria in early diagnosis of these patients which results in their early treatment.

\section{Conclusion}

Fine needle aspiration cytology is an effective and reliable tool in the diagnosis of superficial lymphadenopathy. This may be recommended as the first line of investigation in the diagnosis of lymph node enlargement for patients who may not be able to afford the health cost of surgery and biopsy in our locality. Again, Fine needle aspiration cytology has been proven to be a cheap, easy outpatient procedure with relatively much more economical advantages.

\section{References}

1. Darnal HK, Karim N, Kamini K, Angela K (2005). The Profile of Lymphadenopathy in Adults and Children. Med J Malaysia, 60, 590-8.

2. Singh K, Dubey VK, Khajuria R.Diagnostic accuracy of fine needle aspiration cytology when compared to histopathology. J Cytol 2003; 20: 22-7

3. Tilak V, Dhaded AV, Rain R. Fine needle aspiration cytology of head and neck masses. Indian J Pathol Microbiol 2002; 45: 23-30

4. Bhuyan MAH, Fakir MAY, Hossain ABMT, Huq AHMZ, Gupta S (2008). Role of Fine needle aspiration cytology in the diagnosis of cervical lymphadenopathy.

Bangladesh

$J$

Otorhinolaryngol, 14, 63-5.

5. Ahmad SS, Akhtar S, Akhtar K, Naseem S, Mansoor T (2005). Study of fine needle aspiration cytology in lymphadenopathy with special reference to Acid-fast staining in cases of tuberculosis. JK Science, 7, 1-4.

6. Khan AH, Hayat AS, Baloch GH, et al (2011). Study on the role of fine needle aspiration cytology in cervical lymphadenopathy. World Applied Sci J, 12, 1951-4.

7. Fatima S, Arshad S, Ahmed Z, Hasan SH (2011). Spectrum of Cytological Findings in Patients with Neck LymphadenopathyExperience in a Tertiary Care Hospital in Pakistan. Asian Pac J Cancer Prev, 12, 1873-5.

8. Steel BL, Schwartz MR, Ibrahim R (1995). Fine needle aspiration biopsy in diagnosis of lymphadenopathy in 1,103 patients. Acta Cytolo, 39, 76-81.

9. Mitra S, Ray S, Mitra PK (2011). Fine needle aspiration cytology of supraclavicular lymph nodes: Our experience over a three-year period. $J$ Cytol, 28, 108-10.

10. Khajuria R, Goswami KC, Singh K, Dudey VK (2006). Pattern of lymphadenopathy on fine needle aspiration cytology in Jammu, $J K S c i, 8,145-9$.

11. Hirachand S, Lakhey M, Akhter J, Thapa B (2009). Evaluation of fine needle aspiration cytology of lymph nodes in Kathmandu Medical College, Teaching hospital. Kathmandu Univ Med J, 7, 13942.

12. Wilkinson AR, Mahore SD, Maimoon SR (2012). FNAC in the diagnosis of lymph node malignancies: A simple and sensitive diagnostic tool. Ind J Med Pead Oncol, 33, 21-4.

13. Pandit AA, Candes FP, Khubchandani SR (1987). Fine needle cytology of lymphnodes. J Postgrad Med, 33, 134-6. 
14. Nidhi P, Sapna T, Shalini M, Kumud G (2011). FNAC in tuberculous lymphadenitis: Experience from a tertiary level referral centre. Indian J Tub, 58, 1027.

15. Dhingra V, Misra V, Mishra R, Bhatia R, Singhal M (2010). Fine needle aspiration cytology (FNAC) as a diagnostic tool in Paediatric lymphadenopathy. J Clin Diagn Res, 4, 2452-7.

16. Martin DA, Janes OA, Allen SL, John nd EN. Clinical Oncology 2 Ed. London (UK): Churchill Livingstone Inc. 2000:2620-9 